\title{
Poly(ethylene glycol) and poly(N-vinlypyrrolidone) for improved porcine islet cryopreservation
}

\author{
Dong Xie $^{{ }^{*}}$, Sherly Jules ${ }^{1}$, Yiming Weng ${ }^{1}$, Yuan Zhou ${ }^{1}$, Richard A. Sidner ${ }^{2}$, Mark Pescovitz ${ }^{2}$ \\ ${ }^{1}$ Department of Biomedical Engineering, Purdue School of Engineering and Technology, Indiana University-Purdue University, In- \\ dianapolis, USA \\ ${ }^{2}$ Department of Surgery, School of Medicine, Indiana University, Indianapolis, USA \\ Email: *dxie@iupui.edu
}

Received 11 February 2012; revised 18 March 2012; accepted 28 March 2012

\begin{abstract}
Water-soluble polymers poly(ethylene glycol) (PEG) and poly(N-vinylpyrrolidone) (PVP) were used to study cryopreservation of porcine islets. DMSO was used as control. The effects of polymer purity, molecular weight (MW) and concentration on islet viability were investigated. The results show that both PVP and PEG are good cyroprotectant candidates for islet cryopreservation. The effects of polymer purity and concentration were significant. Increasing concentration significantly increased the islet viability. However, after the concentration reached a certain level, there was no significant difference in viability probably due to increased viscosity of the polymer solution. The effect of polymer MW was not significant. It is concluded that polymers can be a suitable cryoprotectant for porcine islet cryopreservation. The islet viability is polymer concentration-dependent. It seems that PVP is a better cryoprotectant candidate as compared to PEG because the former showed a fast dissolution rate in culture medium and lower viscosity. The polymer concentration at $30 \%$ appears to be the optimal for cryopreservation from the viewpoint of islet viability and medium viscosity.
\end{abstract}

Keywords: Poly (Ethylene Glycol);

Poly(N-Vinylpyrrolidone); Porcine Islet;

Cryopreservation; Cell Viability; Viscosity

\section{INTRODUCTION}

Diabetes mellitus affects $6.3 \%$ of the United States population, ranking as the seventh leading cause of death [1]. There are approximately 171 million patients with diabetes mellitus in the world [1]. It is estimated that this number will increase to 398 million by the year 2025 [1]. Patients with type I diabetes can no longer produce insu-

*Corresponding author. lin in response to glucose in their diet because insulin is synthesized in the islets. Current therapy for patients with type I diabetes includes insulin injection, dietary constraint, exercise, pancreas transplantation and islet transplantation. Islet transplantation is a viable treatment option for patients with type I diabetes. However, there still exist major obstacles to routine use of islet transplantation to treat diabetic patients. These include the severe shortage of human islets and the need to use immunosuppressive drugs to prevent transplant rejection [2]. In addition, the process of islet isolation, purification and transplantation is labor intensive and difficult for routine treatment. It has been suggested that effective cryopreservation techniques to pre-store islets would enhance routine use of islet transplants, even across distant transplant centers. Therefore, islet cryopreservation is an ideal method for long-term storage of islets and is becoming increasingly important as more clinical islet transplants are performed. Islet cryopreservation provides many advantages to a clinical transplantation program, such as long-term storage for banking purpose and transportation of islets. However, one of major loopholes in the process is cryodamage, in which the post-thawing islet cell viability and functionality is highly reduced [3]. Cryodamage occurs mainly during the phase transitions, at the time of freeze-thawing. Dimethylsulfoxide (DMSO) is the most frequently used cryoprotectant in islet cryopreservation [4-8]. However, it is known that use of DMSO in cryopreservation results in about 20\% - 50\% loss in the number and function of islets after thawing $[9,10]$. DMSO is an intracellular cryoprotectant and has deleterious effects on cells at room temperature, as its hydrophobic interactions with proteins can induce their de-stabilization [11]. To avoid osmotic shock, DMSO must be added to the cells in a stepwise manner. Therefore, researchers have been looking for alternatives for DMSO [3,12-14]. The studies include using PEG as a cryoprotectant [12], using hydroxyethyl starch and DMSO as co-cryoprotectants [13], microencapsulation of 
islets with alginate-poly(L-lysine)-alginate before cryopreservation [14], microencapsulation of islets with chitosan-alginate [3], etc. These methods have been found valid at different levels. Use of polymers as a cryoprotectant is believed to increase the viscosity of the medium at low temperature, to inhibit ice crystal growth during cooling or re-warming, not to permeate the cell, and to decrease the freezing point of the medium, thus leading to fast freezing [15].

The objective of this study was to use biocompatible and hydrophilic PEG and PVP as an alternative cryoprotectant to cryopreserve the porcine islets and study the effect of polymer MW and concentration on islet viability.

\section{MATERIALS AND METHODS}

\subsection{Materials and Their Purification}

Dimethylsulfoxide (DMSO) and poly(ethylene glycol) (PEG) including PEG5K, PEG8K and PEG20K with molecular weight (MW) of 5,000, 8,000 and 20,000 Daltons, were received from Sigma-Aldrich Co. (Milwaukee, WI). Poly(N-vinylpyrrolidone) (PVP) including Plasdone C-15 (MW $=10,000)$ or PVP10K and Plasdone C-30 (MW $=59,000)$ or PVP60K were received from Ashland Specialty Ingredients (Wayne, NJ). All the polymers were purified through membrane dialysis against distilled water, followed by freeze-drying and precipitation from alcohol/ether solution. Dialysis is used to remove water-soluble impurities and precipitation is used to remove low MW organic impurities.

\subsection{Porcine Islet Isolation and Purification}

Pancreas was obtained from a local slaughterhouse (Archer's Meat's \& Catering, Greenwood, IN), where pigs weighing typically between 200 - 250 lbs were used as pancreas donors. The pancreas was excised and placed on ice in a container filled with Hanks' balanced salt solution (HBSS) prior to islet isolation. Cold ischemia time did not exceed $3 \mathrm{~h}$. Excess fat and connective tissue surrounding the pancreas were removed. The weight of the cleaned pancreas was noted. After the bile duct was located using a 16, 18 or $20 \mathrm{Ga}$ Teflon catheter, the pancreas was injected with approximately $200 \mathrm{ml}$ of Collagenase diluted in HBSS. Any leaks were clamped or tied off with sutures. The perfused organ was then placed into the lower half of the digestion (Ricordi) chamber with 7 marbles. The re-circulating water bath was typically pre-set at $42^{\circ} \mathrm{C}$ to account for the cold solution added to the chamber. The remaining collagenase solution and HBSS was added to the chamber to fill the system. A screen is placed over the lower half of the chamber and the chamber was sealed shut. The chamber is gently agi- tated once per minute, starting at 5 min into the dissociation. Once the chamber temperature reached $37^{\circ} \mathrm{C}$, the water bath was set to $40^{\circ} \mathrm{C}$ and adjusted with ice to maintain the chamber temperature at $37^{\circ} \mathrm{C}$. A sample of circulating solution was obtained, placed in a Petri dish, stained with Dithizone (DTZ), and viewed under a light microscope for free islets. Once the amount of free islets was greater than $60 \%$, the dissociation was stopped and the circulating solution was collected in bottles filled with HBSS containing $30 \%$ fetal bovine serum. The mixture was then transferred to centrifuge tubes and centrifuged using a centrifuge (Sorvall Legend RT, Global Medical Instrumentation, Inc., Ramsey, MN) at 200g for 4 min. After washing with HBSS containing $10 \%$ fetal bovine serum (FBS) two times, the islet pellet underwent Euro-ficoll solution layering with three different density gradients $(1.037,1.096$, and 1.108) followed by addition of HBSS on the top layer. Tubes were then centrifuged for approximately $21 \mathrm{~min}$ at $100 \mathrm{~g}$ without brake. Pure islets were removed from the interface between 1.037 and 1.096 and re-suspended in media for culturing. $\mathrm{Pu}-$ rity of the islet preparation was assessed by DTZ staining.

\subsection{Islet Culture}

Islet culture was prepared in CMRL 1066 medium (Invitrogen Corporation) supplemented with L-glutamine (5 $\mathrm{ml} / 500 \mathrm{ml})$, Penicillin-Streptomycin $(5 \mathrm{ml} / 500 \mathrm{ml})$, and FBS (heat-inactivated, $50 \mathrm{ml} / 500 \mathrm{ml}$ ). Then islets culture media were incubated overnight at $37^{\circ} \mathrm{C}$ with $5 \% \mathrm{CO}_{2}$ humidified air atmosphere prior to freezing.

\subsection{Freezing-Thawing for Cryopreservation}

\subsubsection{Cryopreservation with DMSO}

The DMSO cryopreservation followed the published procedures with a slight modification [12]. Briefly, after culturing overnight with media of half RPMI 1640 and half McCoy's medium supplemented with $30 \%$ fetal calf serum (FCS) and 1\% mixture of antibiotics, the islets were equilibrated with equal volume of DMSO solution, followed by adding to the tube stepwise and transferring into a 2-mL cryotube. The cryotube was then placed in a programmable cooling machine (Planer Cell Freezer R204, Planer Products Ltd, Sunbury, PA) for freezing, with the programmed procedures shown in Table 1. Immediately after the programmed freezing, the cryotube was stored in liquid nitrogen for $2 \mathrm{~d}$, followed by removing from liquid nitrogen and plunging into a $37^{\circ} \mathrm{C}$ water bath for rapid thawing. After adding CMRL medium, the cryotube was centrifuged for $3 \mathrm{~min}$ at $200 \mathrm{~g}$. The pellet from the cryotube was re-suspended in the culture medium and analyzed for cell viability. 


\subsubsection{Cryopreservation with Polymer}

After complete dissolution of the polymer, the polymer medium solution was filtered to remove potential microorganisms. The islet pellet was then re-suspended into the medium containing polymer (either PEG or PVP), following culturing overnight with media (see 2.4.1) and centrifuging. After well mixing, the islet/polymer medium was transferred into the cryotube, followed by freezing, thawing, and culturing, as the procedures shown in Table 1 . The concentrations of the polymer were $5 \%, 10 \%$, 20\%, 30\% and 40\% (by weight).

\subsection{Islet Viability Test}

Islet cell viability was assessed by microscopic examination using ethidium bromide/fluorescein diacetate staining technique, following incubation or cryopreservation. Briefly, aliquots of islets were placed in a Petri dish and stained with ethidium bromide (5mg/50ml HBSS/HEPES (hydroxyethyl piperazineethanesulfonic acid)) and fluorescein diacetate $(150 \mathrm{mg} / 30 \mathrm{ml}$ acetone). Islets were then observed using a two-color fluorescence microscope. Viable cells were identified with green and dead with red. A percentage (\%) was used to represent the viability. The percentage of viability of the fresh cultured islets before cryopreservation was used as control for each measurement.

\subsection{Viscosity Determination}

The viscosity of the medium containing either DMSO or polymer was determined at $23^{\circ} \mathrm{C}$ using a programmable cone/plate viscometer (RVDV-II + CP, Brookfield Eng. Lab. Inc., Middleboro, MA).

\subsection{Statistical Analysis}

All the viability values were expressed as mean \pm standard deviation (SD). One-way analysis of variance (ANOVA)

Table 1. Programmed procedures for islet cryopreservation.

\begin{tabular}{cl}
\hline $\begin{array}{c}\text { Program } \\
\text { Section \# }\end{array}$ & \multicolumn{1}{c}{ Function } \\
\hline 1 & $\begin{array}{l}\text { Cool chamber to } 0^{\circ} \mathrm{C} \text {, wait until run is started, and then } \\
\text { proceed to } 2\end{array}$ \\
2 & Cool chamber at $2^{\circ} \mathrm{C} / \mathrm{min}$ to $-6^{\circ} \mathrm{C}$ and then proceed to 3 \\
3 & Cool chamber at $25^{\circ} \mathrm{C} / \mathrm{min}$ to $-70^{\circ} \mathrm{C}$ and then proceed to 4 \\
4 & Cool chamber at $10^{\circ} \mathrm{C} / \mathrm{min}$ to $-25^{\circ} \mathrm{C}$ and then proceed to 5 \\
5 & Cool chamber at $0.3^{\circ} \mathrm{C} / \mathrm{min}$ to $-40^{\circ} \mathrm{C}$ and then proceed to 6 \\
6 & Cool chamber at $10^{\circ} \mathrm{C} / \mathrm{min}$ to $-90^{\circ} \mathrm{C}$ and then proceed to 7 \\
7 & Hold chamber at $-90^{\circ} \mathrm{C}$ for 15 min and then proceed to 8 \\
8 & $\begin{array}{l}\text { End program and then place the cryotubes in liquid } \\
\text { nitrogen }\end{array}$ \\
\hline
\end{tabular}

with the post hoc Tukey-Kramer multiple range test was used to determine significant differences of cell viability among the treatments or materials in each group. A level of $\alpha=0.05$ was used for statistical significance.

\section{RESULTS}

Figure 1 shows the effect of cryopreservation on islet viability. The islet viability was in the decreasing order of $100 \%$ for $2 \mathrm{~h}$ at $37^{\circ} \mathrm{C}>76.3 \%$ for 48 h-cryopreservation $>59.3 \%$ for $24 \mathrm{~h}$ at $37^{\circ} \mathrm{C}$, where they were significantly different from each other $(\mathrm{p}<0.05)$.

Figure 2 shows the effect of polymer purity on islet viability. There were statistically significant differences in islet viability between unpurified PEG20K (58.4\%) and purified PEG20K (74.2\%) and between unpurified PVP10K (54.1\%) and purified PVP10K (76.3\%) (p < 0.05).

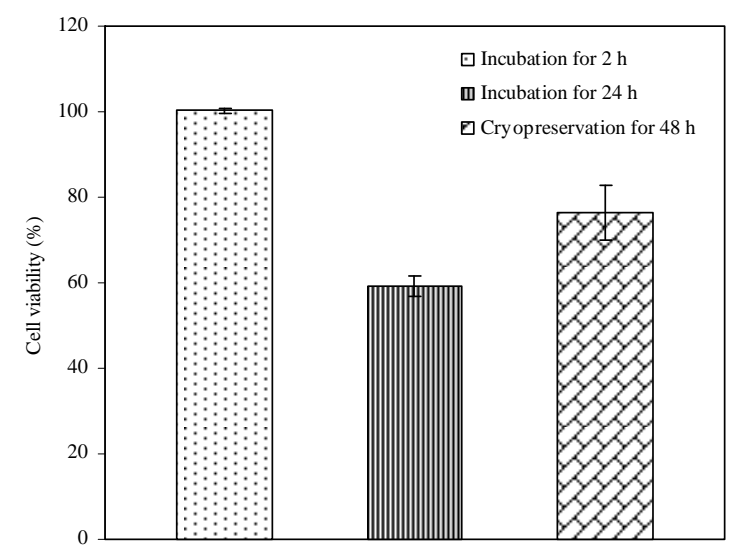

Figure 1. Effect of cryopreservation on islet viability: Comparison among the islet samples incubated at $37^{\circ} \mathrm{C}$ for $2 \mathrm{~h}$, at $37^{\circ} \mathrm{C}$ for $24 \mathrm{~h}$ and cryopreserved for $2 \mathrm{~d}$, after incubating in the $20 \%$ PVP10K-containing medium with half McCoy's and half RPMI 1640 media.

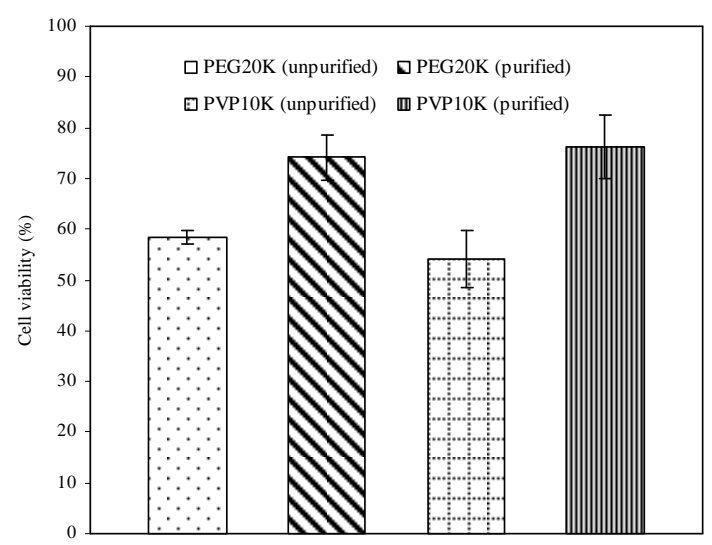

Figure 2. Effect of polymer purity on islet viability: Comparison among unpurified and purified PEG20K as well as PVP10K. PEG20K $=$ PVP10K $=20 \%$. Islet samples in either $20 \%$ PEG20K or PVP10K-containing medium with half McCoy's and half RPMI 1640 media were cryopreserved for $2 \mathrm{~d}$ before viability assessment. 
Table 2 shows the effect of MW of polymer on islet viability. There were no statistically significant differences among PEG5K (58.1\%), PEG8K (60.7\%) and PEG20K (59.7\%) and between PVK10K (63.4\%) and PVK60K (69.7\%) ( $>$ > 0.05).

Figure 3 shows the effect of polymer concentration on islet viability. The islet viability was in the decreasing order of both PEG (Figure 3(a)) and PVP (Figure 3(b)): $30 \%>40 \%>20 \%>10 \%>5 \%$, where $20 \%$, $30 \%$ and

Table 2. Effect of MW of polymer* on islet viability.

\begin{tabular}{ccc}
\hline Material & MW (Dalton) & Cell viability (\%) \\
\hline PEG5K & 5,000 & $58.1(0.54)$ \\
PEG8K & 8,000 & $60.7(0.28)$ \\
PEG20K & 20,000 & $59.7(4.6)$ \\
PVP10K & 10,000 & $63.4(4.1)$ \\
PVP60K & 59,000 & $69.7(0.14)$ \\
\hline
\end{tabular}

${ }^{*}$ Concentration of both PEG and PVP $=10 \%$. Islet samples were cryopreserved for $2 \mathrm{~d}$ before viability assessment.

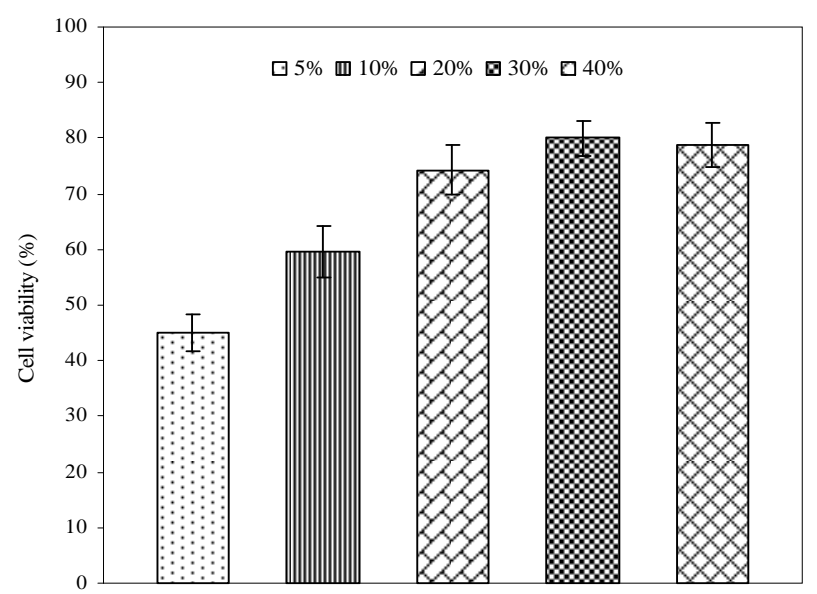

(a)

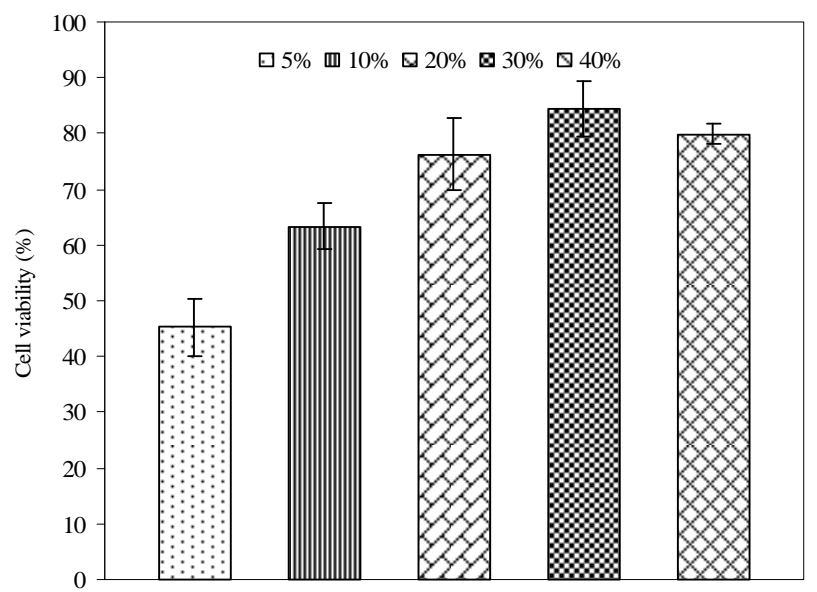

(b)

Figure 3. Effect of polymer concentration on islet viability: (a) PEG20K; (b) PVP10K. Islet samples were cryopreserved for 2 $\mathrm{d}$ before viability assessment.
$40 \%$ were not statistically different from each other $(\mathrm{p}>$ 0.05 ). The viability for $30 \%$ was the highest but the one for $5 \%$ was the lowest.

Table 3 shows the viscosity values of all the polymer/ media solutions used for treating the islets in the study. The viscosity values for PEG were in the range of 1.66 $78.5 \mathrm{cp}$ whereas those for PVP were in the range of 0.68 - 6.53 cp. The viscosity (ср) was in the decreasing order of $40 \%$ PEG20K (78.5) > 30\% PEG20K (38.5) > 20\% PEG20K (20.2) > 40\% PVP10K (6.53) > 10\% PEG20K $(5.25)>30 \%$ PVP10K (4.18) > 10\% PEG8K (2.73) > 10\% PVP60K (2.69) > 20\% PVP10K (2.18) > 5\% PEG20K (2.09) > 10\% PEG5K (1.66) > 10\% PVP10K (0.94) > DMSO (0.72) > 5\% PVP10K (0.68). 40\% PEG20K showed the highest viscosity value but $5 \%$ PVP10K showed the lowest one.

Figure 4 shows the comparison of islet viability among

Table 3. Viscosity values of the polymer solutions with different concentrations used for cryopreservation.

\begin{tabular}{ccc}
\hline Material & Concentration (\%,w/v) & Viscosity (cp) \\
\hline DMSO (78) & 5 & 0.72 \\
PEG20K & 5 & 2.09 \\
PEG20K & 10 & 5.25 \\
PEG20K & 20 & 20.2 \\
PEG20K & 30 & 38.5 \\
PEG20K & 40 & 78.5 \\
PEG5K & 10 & 1.66 \\
PEG8K & 10 & 2.73 \\
PVP10K & 5 & 0.68 \\
PVP10K & 10 & 0.94 \\
PVP10K & 20 & 2.18 \\
PVP10K & 30 & 4.18 \\
PVP10K & 40 & 6.53 \\
PVP60K & 10 & 2.69 \\
\hline
\end{tabular}

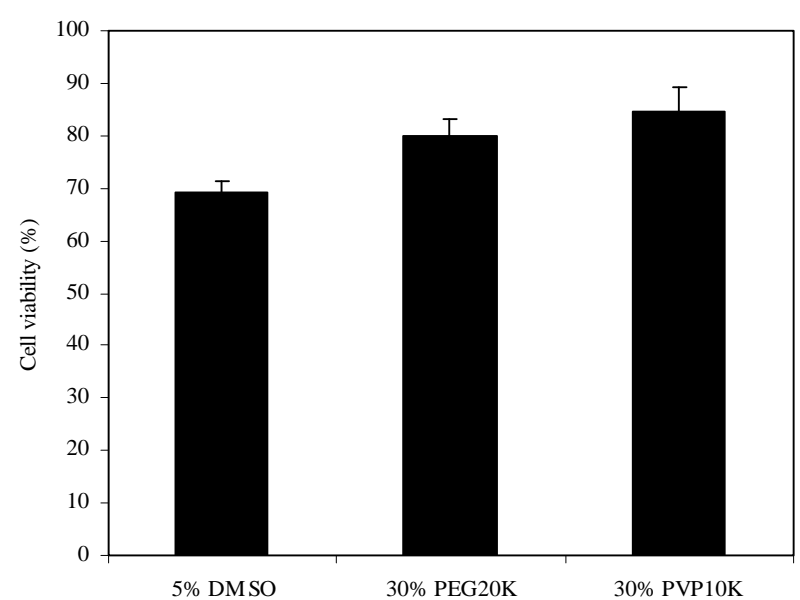

Figure 4. Comparison among DMSO, PEG and PVP: DMSO = $5 \%$, PEG20K $=30 \%$ and PVP10K $=30 \%$. Islet samples were cryopreserved for $2 \mathrm{~d}$ before viability assessment. 
the media containing 5\% DMSO, 30\% PEG20K, and $30 \%$ PVP10K. The islet viability was in the decreasing order of PVP10K (84.5\%) > PEG20K (80.1\%) > DMSO (69.1\%), where PVP10K and PEG20K were not significantly different from each other $(\mathrm{p}<0.05)$.

\section{DISCUSSION}

The result in Figure 1 illustrates that the islets cannot be preserved at $37^{\circ} \mathrm{C}$ or ambient temperature too long because the environmental temperature can accelerate the metabolism of the cells and thus shorten the viability $[5,12]$. However, cryopreservation can stop the unnecessary consumption of the islets and save the energy of the cells [4-8]. That is why the cryoperserved islets showed a higher survival rate than those incubated at $37^{\circ} \mathrm{C}$ for $24 \mathrm{~h}$, although they still showed a lower rate than those incubated for $2 \mathrm{~h}$.

The purity of polymer is very important to islet preservation. It is known that cells are very vulnerable to chemicals. The impurity in unpurified polymer can kill cells. The dialysis of polymer against water can efficiently remove the water-soluble impurity from polymer whereas precipitation using organic solvent/non-solvent is another efficient way to remove the organic impurity. That is why Figure 2 shows significant differences in islet viability between unpurified and purified polymers. In other words, purification is necessary in enhancing biocompatibility of polymers and thus enhancing viability of cells.

Generally speaking, MW of polymer plays an important role in many applications. In this study, however, it seems that MW did not make any contributions to islet viability. No statistically significant differences were found either among PEG with different MW or between PVP with different MW (see Table 2). It has been noted in other studies that islets cryopreserved with high MW compounds show a significantly higher percent of intact islets than those with low MW ones [15]. It is believed that low MW compounds permeate the cells, thus probably perturbing the cytoskeletal architecture and function of the cells through hydrophobic interactions with the intracellular proteins after thawing [11]. It is speculated that among the adversely affected intracellular proteins are key regulatory enzymes of glycolysis, such as phosphofructokinase [11]. As it is already established, glycolysis is the primary event in glucose metabolism, which is linked to glucose stimulation of insulin secretion [16, 17]. Therefore, it is possible that impaired insulin secretion observed after cryopreservation with the low molecular weight cryoprotectants may be linked to their adverse effects on glycolytic enzymes of the beta cells $[16,17]$.

Figure 3 shows the effect of polymer concentration on islet viability. We have noticed that the effect of polymer concentration is not directly proportional to islet viability. With increase of polymer concentration, islet viability significantly increased from the concentration of $5 \%$ to $30 \%$ and then decreased from $30 \%$ to $40 \%$, although there were no statistically significant differences among $20 \%, 30 \%$ and $40 \%$ for either polymer. It seems that islet viability is polymer-concentration dependent at a concentration below $30 \%$. It is suggested that polymer with a concentration of $30 \%$ may be the optimal for cryopreservation for both polymers.

In the study, we noticed that the viscosity value of the polymer solution is correlated to the concentration of the polymer. It is known that the higher the polymer concentration, the higher the viscosity. Table 3 shows the viscosity values of all the polymer/medium solutions used for treating the islets in the study. The viscosity values (1.66 - 78.5 cp) for PEG were much higher than those (0.68 - 6.53) for PVP. Apparently, PVP is more watercompatible or water-friendly polymer than PEG. PVP has been used as a blood plasma extender for trauma victims after the 1950's, due to its biocompatibility and water solubility [18]. At the same concentration, PVP showed a much lower viscosity value than PEG. For example, at the concentration of $40 \%$, the viscosity for PVP was 6.53 but 78.5 for PEG, although the latter had a higher MW $(20 \mathrm{~K})$ than the former $(10 \mathrm{~K})$. At a concentration of $10 \%$, high MW of PVP60K showed a low viscosity (2.69 cp), as compared to PVP10K (0.94 cp), PEG8K (2.73 cp). During the study, we also noticed that PEG with a higher concentration was very difficult to dissolve in the medium and required a much longer time to be incorporated into the medium. The formed PEGcontaining medium was also hard to be filtered for sterilization before mixing with the islets. On the contrary, PVP was very easy and quick to dissolve in the medium even at the concentration of $40 \%$. A lower solution viscosity of polymer can help quickly pass the polymer solution through a microbial filter for filtering bacteria out, which makes the sterilization process simpler. Therefore, from the viewpoint of either dissolution rate of polymer or filtration of polymer for sterilization, PVP is a better candidate than PEG. The results also indicate that using $30 \%$ of either PEG or PVP polymers to cryopreserve the islets might be the optimal concentration because this concentration not only shows the highest islet viability but also exhibits a relatively low viscosity. The results also indicate that PVP is a better cryoprotectant than PEG, because it shows a very low viscosity even at a very high $\mathrm{MW}$ of $60 \mathrm{~K}$.

Finally, we compared the islet viability between cryopreservation with low MW DMSO and with polymers (PEG and PVP). As shown in Figure 4, 30\% PVP10K and 30\% PEG20K showed significantly higher islet viability values than DMSO. This indicates that polymers 
are an excellent potential candidate as a cryoprotectant for islet preservation. Since DMSO has potential deleterious effects such as tissue damage and/or alteration of islet function [11], the polymers we have investigated in this study may eventually replace DMSO as a safe and efficient cyroprotectant for islet and possibly other cell preservation. It is known that polymers can protect the cells from forming harmful ice crystals during cooling and re-warming $[3,12,14]$. Polymers can increase the viscosity of the culture medium at low temperatures, thereby decreasing the freezing point of the medium and thus causing rapid freezing [15]. It is also believed that polymers do not permeate the cells due to their huge size and thus do not interact with intracellular proteins and destabilize the proteins, unlike DMSO [15].

\section{CONCLUSIONS}

This study reports using water-soluble polymers PEG and PVP to cryopreserve porcine islets. The results show that both PEG and PVP are excellent cyroprotectant candidates for islet cryopreservation. The effects of purity and concentration of polymers are significant. The polymers used for islet cryopreservation need to be purified. Increasing concentration significantly increased the islet viability. However, after the concentration reached a certain level, there was no significant difference in viability probably due to increased viscosity of the polymer solution. It is concluded that polymers can be a suitable cryoprotectant for porcine islet cryopreservation. The islet viability is polymer concentration-dependent. It seems that PVP is a better cryoprotectant candidate as compared to PEG because the former showed a fast dissolution rate in culture medium and lower viscosity. Both PEG and PVP with a concentration of $30 \%$ appear to perform the best from the viewpoint of islet viability and medium viscosity. Future work will include functionality determination of the optimal polymer formulation, viability and functionality determination of long-term cryopreservation, and cryopreservation applied for human islets and islets for other animals.

\section{ACKNOWLEDGEMENTS}

This paper is specially published for memorizing Dr. Mark Pescovitz world-renowned organ surgeon and scientist for pancreas transplantation, who died in a tragic accident in 2010.

\section{REFERENCES}

[1] Bacha, F., Lee, S., Gungor, N. and Arslanian, S.A. (2010) From pre-diabetes to type 2 diabetes in obese youth Pathophysiological characteristics along the spectrum of glucose dysregulation. Diabetes Care, 33, 2225-2231. doi:10.2337/dc10-0004

[2] Biancone, L. and Ricordi, C. (2002) Pancreatic islet transplantation: An update. Cell Transplant, 11, 309-311.

[3] Hardikar, A.A., Risbud, M.V. and Bhonde, R.R. (2000) Improved post-cryopreservation recovery following encapsulation of islets in chitosan-alginate microcapsules. Transplantation Proceedings, 32, 824-825. doi:10.1016/S0041-1345(00)00995-7

[4] Cattral, M.S., Warnock, G.L., Kneteman, N.M., Halloran, P.F. and Rajotte, R.V. (1993) The effect of cryopreservation on the survival and MHC antigen expression of murine islet allografts. Transplantation, 55, 159-163. doi:10.1097/00007890-199301000-00029

[5] Charles, K., Harland, R.C., Ching, D. and Opara, E.C. (2000) Storage and microencapsulation of islets for transplantation. Cell Transplantation, 9, 33-38.

[6] Janjic, D., Andereggen, E., Deng, S., Bartley, C., Buhler, L., Morel, P. and Wollheim, C.B. (1996) Improved insulin secretion of cryopreserved human islets by antioxidant treatment. Pancreas, 13, 166-172. doi:10.1097/00006676-199608000-00008

[7] Langer, S., Lau, D., Eckhardt, T., Jahr, H., Brandhorst, H., Brandhorst, D., Hering, B.J., Federlin, K. and Bretzel, R.G. (1999) Viability and recovery of frozen-thawed human islets and in vivo quality control by xenotransplantation. Journal of Molecular Medicine, 77, 172-174. doi:10.1007/s001090050330

[8] Miyamoto, M., Kenmochi, T., Nakagawa, Y., Une, S., Moldovan, S., Atiya, A., Benhamou, P.Y., Brunicardi, F.C., Ohyanagi, H. and Mullen, Y. (1995) Immunogenicity of cryopreserved human islets. Transplantation Proceedings, 27, 3406-3408.

[9] Rich, S.J., Swift, S., Thirdborough, S.M., Rumford, G., James, R.F. and London, N.J. (1993) Cryopreservation of rat islets of Langerhans: A comparison of two techniques. Cryobiology, 30, 407-412. doi:10.1006/cryo.1993.1040

[10] Rich, S.J., Swift, S., Thirdborough, S.M., James, R.F. and London, N.J. (1994) Islet cryopreservation: A detailed study of total functional losses. Transplantation Proceedings, 26, 823-824.

[11] Arakawa, T., Carpenter, J.F., Kita, Y.A. and Crowe, J.H. (1990) The basis for toxicity of certain cryoprotectants: A hypothesis. Cryobiology, 27, 401-415. doi:10.1016/0011-2240(90)90017-X

[12] Monroy, B., Honiger, J., Darquy, S. and Reach, G. (1997). Use of polyethyleneglycol for porcine islet cryopreservation. Cell Transplantation, 6, 613-621. doi:10.1016/S0963-6897(97)00097-3

[13] Maruyama, M., Kenmochi, T., Sakamoto, K., Arita, S., Iwashita, C. and Kashiwabara, H. (2004) Simplified method for cryopreservation of islets using hydroxyethyl starch and dimethyl sulfoxide as croprotectants. Transplantation Proceedings, 36, 1133-1134. doi:10.1016/j.transproceed.2004.04.016

[14] Inaba, K., Zhou, D., Yang, B., Vacek, I. and Sun, A.M. (1996) Normalization of diabetes by xenotransplantation of cryopreserved microencapsulated pancreatic islets. Application of a new strategy in islet banking. Transplantation, 61, 175-179. doi:10.1097/00007890-199601270-00001 
[15] O’Neil, L., Paynter, S.J. and Fuller, B.J. (1997) Vitrification of mature mouse oocytes: Improved results following addition of polyethylene glycol to a dimethyl sulfoxide solution. Cryobiology, 34, 295-301. doi:10.1006/cryo.1997.2007

[16] Meglasson, M.D. and Matschinsky, F.M. (1984) New perspectives on pancreatic islet glucokinase. American Journal of Physiology, 246, E1-E13.
[17] Murata, T., Miwa, I., Toyoda, Y. and Okuda, J. (1993) Inhibition of glucose-induced insulin secretion through inactivation of glucokinase by glyceraldehyde. Diabetes, 42, 1003-1009. doi:10.2337/diabetes.42.7.1003

[18] Haaf, F., Sanner, A. and Straub, F. (1985) Polymers of N-vinylpyrrolidone: Synthesis, characterization and uses. Polymer Journal, 17, 143-152. doi:10.1295/polymj.17.143 